\title{
Multicellular Spheroids Formation and Recovery in Microfluidics-generated Thermoresponsive Microgel Droplets
}

\author{
X. Cui ${ }^{\text {a }}$, Y. Liu ${ }^{\text {b }}$, Y. Hartanto a ${ }^{\text {, J. Bi }}{ }^{\text {a }}$, S. Dai ${ }^{\text {a }, ~ H . ~ Z h a n g ~}{ }^{\text {a,* }}$ \\ a School of Chemical Engineering, The University of Adelaide, Adelaide, SA 5005, Australia \\ ${ }^{\mathrm{b}}$ School of Biochemical Engineering, East China University of Science and Technology, Shanghai, China
}

\section{A R T I C L E I N F O}

\section{Article history:}

Received 10 August 2016

Received in revised form 24 August 2016

Accepted 11 September 2016

Available online 21 September 2016

\section{Keywords:}

Biomaterials

Thermal response microgels

Droplet

Microfluidics

Multicellular spheroids

\begin{abstract}
A B S T R A C T
Multicellular spheroids (MCSs) have a unique structure to represent the in vivo tissues and they have many potential applications, such as in drug screening and evaluation. Traditional methods of fabricating MCSs are incapable of controlling the MCS size and size distribution. For MCS formation in the ion- or photo-crosslinked hydrogel, recovery of MCSs from these hydrogels may need strong chemicals or enzymes to break the crosslinked network for release of MCSs. We are the first to develop a microfluidic approach to encapsulate Hela cells in thermoresponsive microgel-based droplets to generate Hela MCSs. The microgel network provided a physical scaffold for cells so that cell aggregates formed in a biomimicking condition. Due to the thermal reversibility of microgels, MCSs were released and harvested from the microgels droplets by simply cooling the droplets down to room temperature. This approach may open a new door for generating uniform-sized MCSs.
\end{abstract}

(C) 2016 Elsevier B.V. This is an open access article under the CC BY-NC-ND license

(http://creativecommons.org/licenses/by-nc-nd/4.0/).
Most cells in the human body are living in a three-dimensional (3D) environment through interacting with neighboring cells and extracellular matrices (ECM) which are missing in traditional two dimensional monolayer culture [1]. Cell-cell and cell-ECM interactions have a great impact on many biological activities such as homeostasis and cell signaling through functional junctions between cells [2]. The 3D cell culture model based on multicellular spheroids (MCSs) is a powerful tool to recapitulate the cell-cell and cell-matrix interactions that have been found in vivo to preserve cellular viability, functionality and phenotype [3], and the MCSs serve a building bridge to connect the two-dimensional cell culture and animal-based studies [4]. Hence, a myriad of methods have been developed for fabrication of MCSs. Conventional methods such as the hanging drop [5], the gyratory method [6], non-adhesive culture [7], micro-fabricated confined culture [8], accelerated aggregate-forming method [9] as well as liquid overlay culture [10] have been explored. Among them, the micro-fabrication technology has been recently pursued due to manipulatable sizes and shapes of MCSs in the confined environment. Microarrays [11], microwells [12] and microfluidic devices [13] are designed to seed a high cell density in a small cavity so that cells aggregate in the cavity to form MCSs. However, in this method, cells tend to settle in the bottom of the cavity due to gravity and the formed MCSs are unable to easily recover from the microfabrication devices.

\footnotetext{
* Corresponding author.

E-mail address: hu.zhang@adelaide.edu.au (H. Zhang).
}

Cell encapsulation in microfluidic channels is achieved by mixing the cell-laden hydrogels in one channel with crosslinking agents from another channel(s). This approach allows rapid formation, high-throughput [14] and miniaturized bioreactors for cell culture [15]. To date, hydrogels like alginate, agarose, gelatin and poly (ethylene glycol) [16-19], as well as double emulsion [20] have been attempted to encapsulate cells in these hydrogels. Very few attempts are reported to culture cells in the crosslinked gel for a long term. Since the polymers are chemical crosslinked, potent chemicals may be required to break the shell to release MCSs [21]. For the double emulsion encapsulation since the middle layer between cells and the culture medium is an oil phase, the oxygen and nutrient diffusion rate is low, and this becomes the constraints for MCS growth. We are the first group to propose a dropletbased microfluidics system to form and release spheroids using thermal-responsive synthetic polymers. Different from natural polymers, this unique thermoresponsive material can release MCSs easily after long-term culture without introduction of toxic chemicals. In addition, through copolymerizing different co-monomers, the properties of the synthesized polymers can be tuned for different types of cells.

As described in Fig. 1, HeLa cells were encapsulated inside the Poly (N-Isopropylacrylamide-co-acrylic acid) (P(NIAM-AA)) microgel based droplets. $\mathrm{P}(\mathrm{NIPAM}-\mathrm{AA})$ has a unique amphiphilic structure so the microgel network is thermally reversible, which was confirmed from the temperature dependence of its hydrodynamic size (Fig. S1) and elastic/viscous modulus (Fig. S2). The SEM image (Fig. S3) shows a porous structure which not only provides the physical support for 
(c)

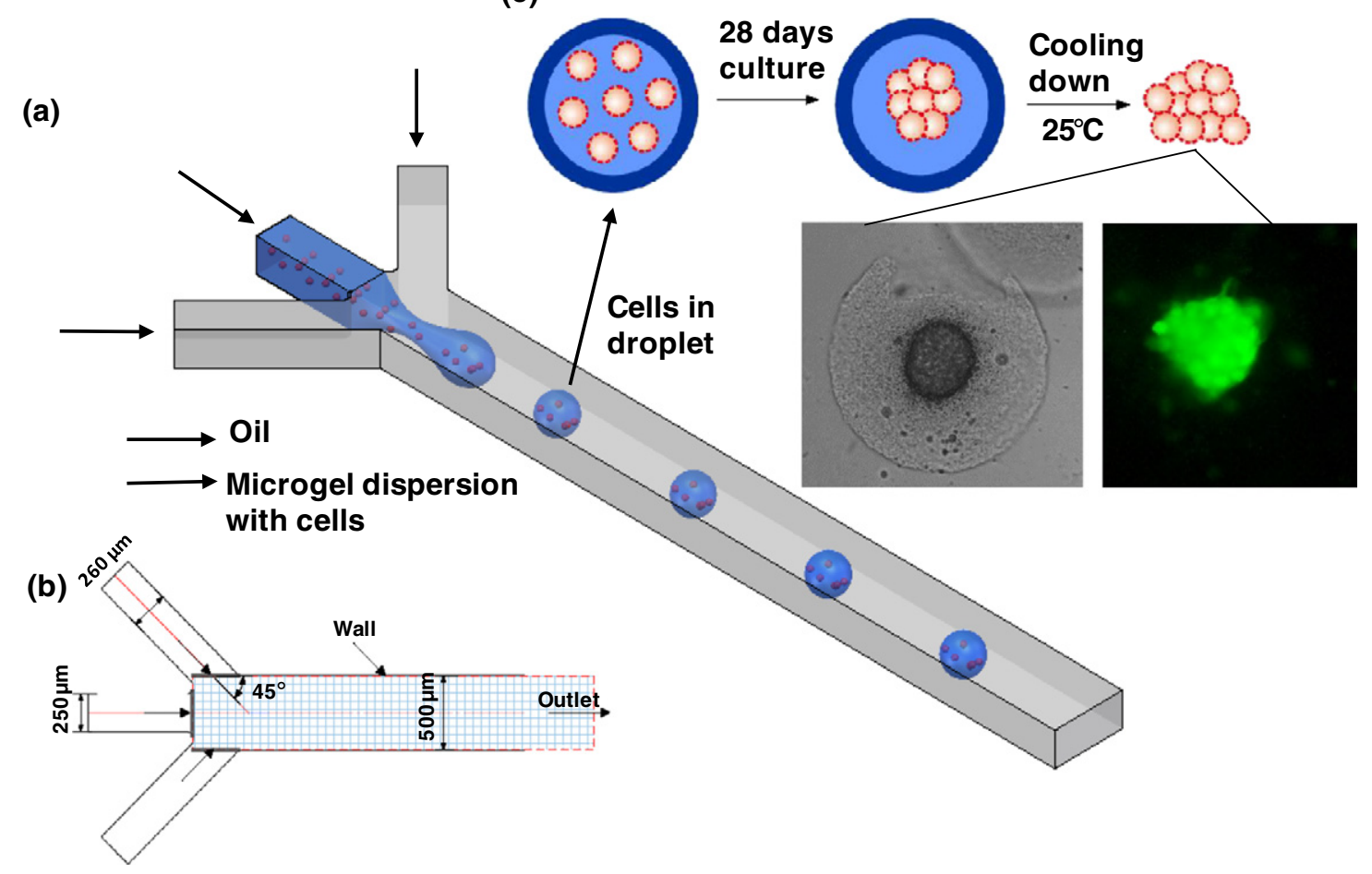

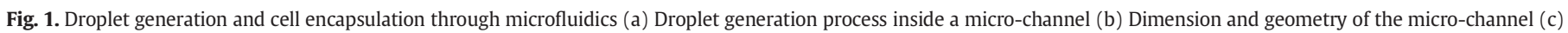
Multicellular spheroids formation inside the droplet.

embedding cells in a 3D microenvironment but also allows sufficient oxygen and nutrient transportation.

The cell-laden P(NIPAM-AA) microgel aqueous solution was squeezed into individual droplets by the oil phase from two side channels. The polydimethylsiloxane (PDMS) microfluidic microchannel was fabricated using the soft-lithography technique. The PDMS microchannel was designed as a flow-focusing device, which was composed of one water channel in the middle and two oil channels at both sides to produce the immiscible interfaces when the two flows were focused at the intersection. Two streams of continuous oil phase flowed through two sides and squeezed the forefront stream of the dispersed fluid into droplets. The Lattice Boltzmann model was applied to two immiscible phases for simulation of the droplet formation within the microchannel, and the optimized flow rate to generate discrete droplets for both phases was used for the following experiments (Fig. S4). Compared to experimental data, the LBM simulation can accurately predict the flow phenomenon within microchannel. When the flowrate ratio is at 1:1 between the dispersed phase and the continuous phase, jetting flow was observed without droplet generation. At a flowrate ratio of 1:4, the majority (more than $40 \%$ ) of the droplets have the size around $260 \mu \mathrm{m}$. More than $80 \%$ of the droplets are in the range between 250 and $350 \mu \mathrm{m}$. When increasing the continuous phase flow rate, at flowrate ratio of 1:6, the droplet size is around $100 \mu \mathrm{m}$. In order to obtain reasonable size of droplets to culture MCSs, the flowrate ratio at 1:4 was chosen for future experiments.

After droplets were generated inside the microchannel and collected at the exit, the droplets were heated up to $37{ }^{\circ} \mathrm{C}$, and their colour changed from transparent (Fig. 2a) into white which is shown at Fig. $2 \mathrm{~b}$. When the temperature of the microgels within the droplets was above the volume phase transition temperature of the microgels (VPTT, around $32{ }^{\circ} \mathrm{C}$ from our previous report [22]), the microgels became hydrophobic from hydrophilic due to the presence of $\mathrm{N}$ isopropylacrylamide. In additional, due to hydrophobic attractions and electrostatic repulsions, physical gel was formed [22]. The physical gel presented a white cloudy colour, which is shown darkness under an optical microscope. The size of the droplet slightly decreased during the heating up process since the microgels shrank due to loss of surrounding water molecules. After washing the microgels with prewarmed PBS buffer $\left(37^{\circ} \mathrm{C}\right)$ to remove the oil residue, the droplets were transferred into a pre-warmed culture medium. The physical gel was able to maintain its spherical shape as shown in Fig.2c, since at $37^{\circ} \mathrm{C}$, the hydrophobic nature of the microgel prevented water from
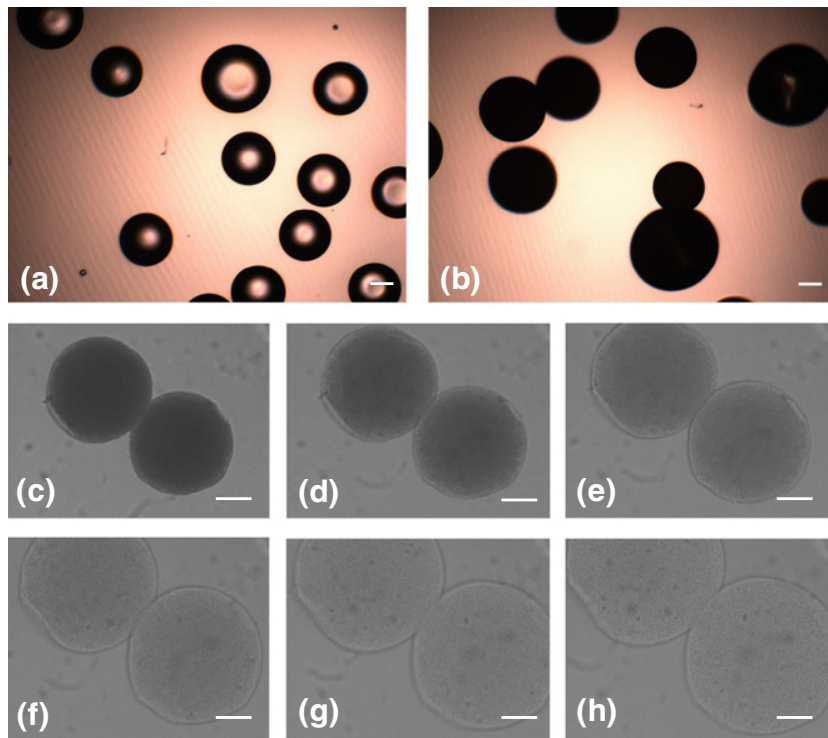

Fig. 2. Droplets collection and thermal response for elevating and decreasing temperature. (a) Microgel solution droplets collection at room temperature in the oil. The dark edge of droplets is seen due to light reflection between oil and water. (b) The changes of hydrophobicity of droplets from hydrophilic to hydrophobic at $37{ }^{\circ} \mathrm{C}$ in oil. Opaque hydrophobic droplets show complete darkness. (e) to (h) Droplets in culture media cool down to temperature every $2 \mathrm{~min}$. Scale bar is $100 \mu \mathrm{m}$. 
entering into its inner structure. Fig. 2d-h demonstrate the collapse process of the microgel structure every 2 min when the temperature reduces to the room temperature. During the first $6 \mathrm{~min}$, the colour of the droplet gradually changed into transparent and the droplets were able to maintain their size and shape. After $6 \mathrm{~min}$, the droplets started to lose their shape, and the size of the droplets expanded, which indicated microgels were dispersed into water.

We hypothesized that cells are encapsulated into the uniform-sized droplets generated from the microchannel, and then the droplets gel at $37^{\circ} \mathrm{C}$ and disperse in the cell culture medium for cell growth and cellular spheroid formation, finally the cellular spheroids are released from the droplets after the temperature reduces to the room temperature for harvest, as shown in Fig. 1. Comparing with other previous reports using droplet encapsulation of cells [19,21], in which MSCs are unable to release from droplets without strong or toxic chemicals. We encapsulated HeLa cells inside the microgel-containing droplets. After suspending droplets in the cell culture medium for the pre-set days, we collected the droplets and cooled down to the room temperature to harvest the MCSs. We found out that it took $20 \mathrm{~min}$ to dissolve the droplets and release the MCSs. Fig. 3 shows the HeLa MCS formation process inside the droplets for up to 28 days. The porous structure inside the droplet provided a 3D structure to support cell growth due to sufficient nutrient, oxygen and biowaste transportation (Fig. S3). At day 7 , cells proliferated and aggregated to form a few cell clusters in each droplet, and the cell cluster size varied depending on the initial cell distribution within the droplets, which was similar to cluster formation in the 3D scaffold [23] in comparison with single individual cells at day 1 as shown in Fig. S5. After continuing cell culture for another 7 days (Fig.3b), the size of the big cluster increased to $40-50 \mu \mathrm{m}$ while a few small clusters scattered around the big clusters. The loose structure indicates the first step of multicellular spheroid formation where cells are drawn together due to the combination of ECM fibres with multiple RGD motifs [24]. At day 21 (Fig. 3c), smaller clusters aggregated into the big cluster to have a size of $50 \mu \mathrm{m}$ and very few smaller clusters were observed within the droplet. At day 28 , the edge of the big cluster become smoother which indicates spheroid compaction stage was reached [24] where the homophilic cadherin-cadherin binding and microfilaments of cells become localized along the cell. Due to controlled microenvironment provided from the droplet, the spheroid size can be manipulated by adjusting the droplet size, initial cell density and cell culture
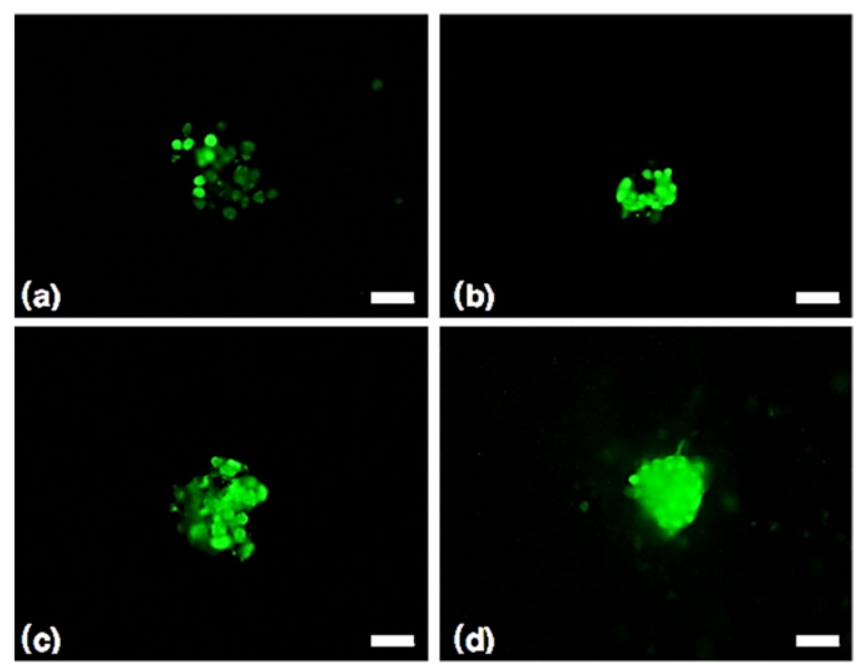

Fig. 4. Live/dead images of the MCSs inside the droplets at (a) day 7, (b) day 14, (c) day 21, (d) day 28. Scale bar is $50 \mu \mathrm{m}$.

duration. Compared with the normal HeLa cluster size around $250 \mu \mathrm{m}$ and the size variations of $50 \mu \mathrm{m}$ in the conventional culture condition, this approach can provide more uniform-sized multicellular spheroids.

To further investigate the cluster morphology, live/dead cells images were taken by staining cells with the Live/Dead cell kit. Fig. 4 shows the cluster formation process from the encapsulated cells within a droplet. This approach was benign to cells and most cells were still green up to 28 day culture, which means cells have a high viability. At day 7, within the droplet, smaller clusters started to form (Fig. 4a), and these clusters were drawn together due to their extracellular matrices. After another 7-day culture (Fig. 4b), small clusters aggregated to form a loose structure, and a few single cells or small clusters were observed around the loose structure. At day 21 (Fig. 4c) the loose structure started the compaction process, but the cavities were seen inside the structure. After 28 day culture (Fig. 4d), mature spheroids were obtained with a spherical shape and a very compact structure was observed.

In this hydrogel-based droplet microenvironment, Hela cells are confined in the given space and aggregated to form the spheroids.
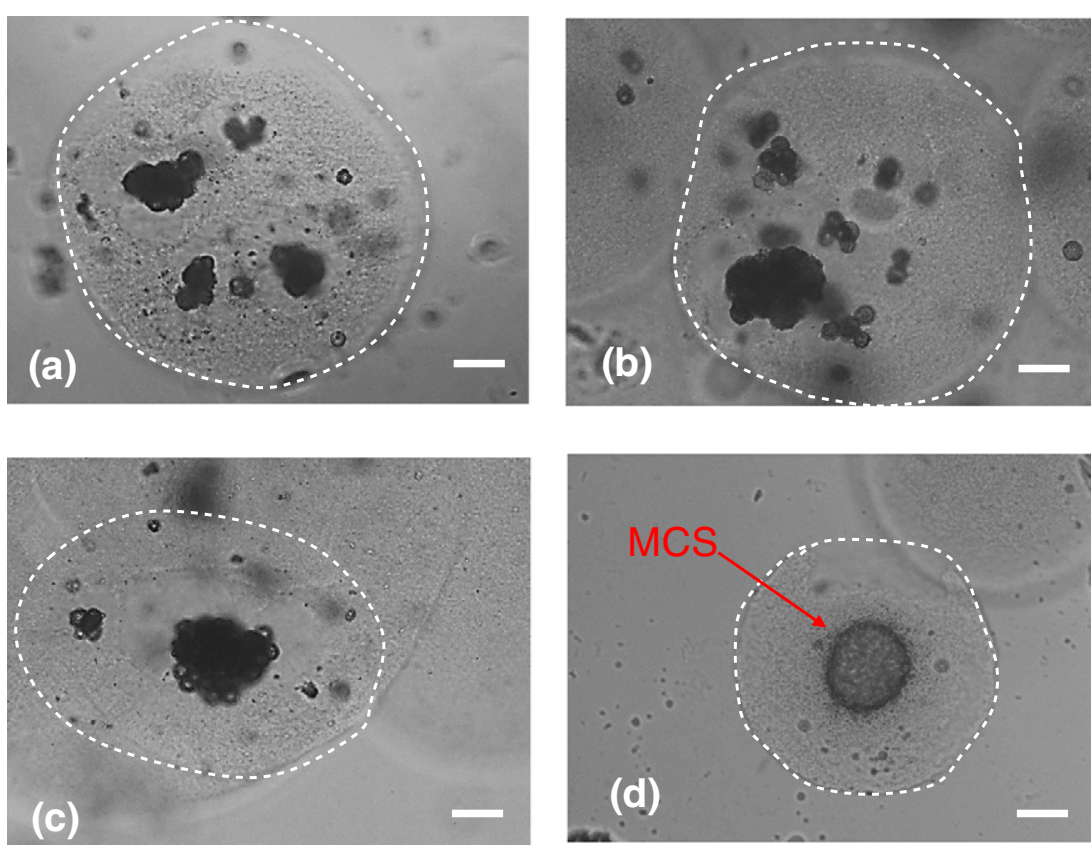

Fig. 3. MCSs formation inside the droplets at (a) day 7, (b) day 14, (c) day21, (d) day 28 . Scale bar is $50 \mu$ m. Dash line is droplet boundary. 
Meanwhile the nutrients and wastes are transferred to the cell clusters to maintain cellular activities. Importantly, the spheroids are harvested simply by reversing the temperature to the room temperature without physical or chemical damage. Therefore, the full recoverability and the 3D controlled microenvironment render our approach very promising for size-controllable multicellular spheroid formation.

The microfluidic channels were made in the ANFF SA node at The University of South Australia and we acknowledge technical supports from the team at the node. XC would like to acknowledge the divisional scholarship given by the University of Adelaide. $\mathrm{HZ}$ thanks the financial support from ARC Discovery Project (DP160104632) and The Medical Advancement Without Animal (MAWA) Trust.

\section{Appendix A. Supplementary data}

Supplementary data to this article can be found online at http://dx. doi.org/10.1016/j.colcom.2016.09.001.

\section{References}

[1] F. Pampaloni, E.G. Reynaud, E.H.K. Stelzer, The third dimension bridges the gap between cell culture and live tissue, Nat. Rev. Mol. Cell Biol. 8 (2007) 839-845.

[2] M.W. Bosenberg, J. Massagué, Juxtacrine cell signaling molecules, Curr. Opin. Cell Biol. 5 (1993) 832-838

[3] H.-F. Lu, K.-N. Chua, P.-C. Zhang, W.-S. Lim, S. Ramakrishna, K.W. Leong, H.-Q. Mao, Three-dimensional co-culture of rat hepatocyte spheroids and NIH/3T3 fibroblasts enhances hepatocyte functional maintenance, Acta Biomater. 1 (2005) 399-410.

[4] Y. Wang, J. Wang, Mixed hydrogel bead-based tumor spheroid formation and anticancer drug testing, Analyst 139 (2014) 2449-2458.

[5] S.L. Nyberg, J. Hardin, B. Amiot, U.A. Argikar, R.P. Remmel, P. Rinaldo, Rapid, largescale formation of porcine hepatocyte spheroids in a novel spheroid reservoir bioartificial liver, Liver Transpl. 11 (2005) 901-910.

[6] R.L. Carpenedo, C.Y. Sargent, T.C. McDevitt, Rotary suspension culture enhances the efficiency, yield, and homogeneity of embryoid body differentiation, Stem Cells 25 (2007) 2224-2234.

[7] J.M. Yuhas, A.P. Li, A.O. Martinez, A.J. Ladman, A simplified method for production and growth of multicellular tumor spheroids, Cancer Res. 37 (1977) 3639-3643.

[8] A.P. Napolitano, P. Chai, D.M. Dean, J.R. Morgan, Dynamics of the self-assembly of complex cellular aggregates on micromolded nonadhesive hydrogels, Tissue Eng. 13 (2007) 2087-2094.
[9] M. Kamihira, K. Yamada, R. Hamamoto, S. Iijima, Spheroid formation of hepatocytes using synthetic polymer, Ann. N. Y. Acad. Sci. 831 (1997) 398-407.

[10] S. Sakai, S. Ito, K. Kawakami, Calcium alginate microcapsules with spherical liquid cores templated by gelatin microparticles for mass production of multicellular spheroids, Acta Biomater. 6 (2010) 3132-3137.

[11] M.-Y. Lee, R.A. Kumar, S.M. Sukumaran, M.G. Hogg, D.S. Clark, J.S. Dordick, Three-dimensional cellular microarray for high-throughput toxicology assays, Proc. Natl. Acad. Sci. 105 (2008) 59-63.

[12] K. HoáLee, D. YoonáNo, J. HeeáRyoo, S. FungáWong, Diffusion-mediated in situ alginate encapsulation of cell spheroids using microscale concave well and nanoporous membrane, Lab Chip 11 (2011) 1168-1173.

[13] M.C. Chen, M. Gupta, K.C. Cheung, Alginate-based microfluidic system for tumor spheroid formation and anticancer agent screening, Biomed. Microdevices 12 (2010) 647-654.

[14] D. Velasco, E. Tumarkin, E. Kumacheva, Microfluidic encapsulation of cells in polymer microgels, Small 8 (2012) 1633-1642.

[15] M. Serra, C. Correia, R. Malpique, C. Brito, J. Jensen, P. Bjorquist, M.J. Carrondo, P.M. Alves, Microencapsulation technology: a powerful tool for integrating expansion and cryopreservation of human embryonic stem cells, PLoS One 6 (2011), e23212.

[16] E. Tumarkin, L. Tzadu, E. Csaszar, M. Seo, H. Zhang, A. Lee, R. Peerani, K. Purpura, P.W. Zandstra, E. Kumacheva, High-throughput combinatorial cell co-culture using microfluidics, Integr. Biol. 3 (2011) 653-662.

[17] S. Sakai, S. Ito, H. Inagaki, K. Hirose, T. Matsuyama, M. Taya, K. Kawakami, Cellenclosing gelatin-based microcapsule production for tissue engineering using a microfluidic flow-focusing system, Biomicrofluidics 5 (2011) 013402

[18] A. Kumachev, J. Greener, E. Tumarkin, E. Eiser, P.W. Zandstra, E. Kumacheva, Highthroughput generation of hydrogel microbeads with varying elasticity for cell encapsulation, Biomaterials 32 (2011) 1477-1483.

[19] S. Utech, R. Prodanovic, A.S. Mao, R. Ostafe, D.J. Mooney, D.A. Weitz, Microfluidic generation of monodisperse, structurally homogeneous alginate microgels for cell encapsulation and 3D cell culture, Adv. Healthcare Mater. 4 (2015) 1628-1633.

[20] H.F. Chan, Y. Zhang, Y.-P. Ho, Y.-L. Chiu, Y. Jung, K.W. Leong, Rapid formation of multicellular spheroids in double-emulsion droplets with controllable microenvironment, Scientific Reports, 3, 2013.

[21] S. Yoon, J.A. Kim, S.H. Lee, M. Kim, T.H. Park, Droplet-based microfluidic system to form and separate multicellular spheroids using magnetic nanoparticles, Lab Chip 13 (2013) 1522-1528.

[22] Z. Shen, J. Bi, B. Shi, D. Nguyen, C.J. Xian, H. Zhang, S. Dai, Exploring thermal reversible hydrogels for stem cell expansion in three-dimensions, Soft Matter 8 (2012) $7250-7257$.

[23] Y. Lei, D.V. Schaffer, A fully defined and scalable 3D culture system for human pluripotent stem cell expansion and differentiationProceedings of the National Academy of Sciences 2013, pp. E5039-E5048.

[24] R.Z. Lin, H.Y. Chang, Recent advances in three-dimensional multicellular spheroid culture for biomedical research, Biotechnol. J. 3 (2008) 1172-1184. 